\title{
Double-stimuli Responsive O/W Emulsion Gel Based on a Novel Amidoamine Surfactant
}

\author{
Clara Morita ${ }^{1,2}$, Hiroki Sugimoto ${ }^{1}$, Yoshiro Imura ${ }^{1,2}$ and Takeshi Kawai ${ }^{1 *}$ \\ ${ }^{1}$ Department of Industrial Chemistry, Tokyo University of Science (1-3 Kagurazaka, Shinjuku-ku, Tokyo 162-8601, JAPAN) \\ ${ }^{2}$ The Japan Society for the Promotion of Science (JSPS) (8 Ichibancho, Chiyoda-ku, Tokyo 102-8471, JAPAN)
}

\begin{abstract}
A heat-induced $\mathrm{O} / \mathrm{W}$ emulsion gel that undergoes a phase transition from sol to gel on heating was formed from the addition of aqueous $\mathrm{HCl}$ to a toluene solution of a long-chain amidoamine derivative (C18AA). The heat induced $\mathrm{O} / \mathrm{W}$ emulsions are highly sensitive to temperature, and the sol-gel transition temperature could be simply controlled by adjusting the C18AA concentration. Interestingly, the sol-gel transition of the $\mathrm{O} / \mathrm{W}$ emulsions was also very sensitive to $\mathrm{pH}$. Thus, we have successfully prepared a novel double-stimuli responsive gel based on $\mathrm{O} / \mathrm{W}$ emulsions consisting of C18AA and $\mathrm{HCl}$.
\end{abstract}

Key words: gel, stimuli-responsive material, $\mathrm{pH}$, emulsion, surfactant

\section{INTRODUCTION}

In recent years, stimuli-responsive materials have been extensively studied because of their potential application in pharmaceuticals, micromachines, and pollutant removal ${ }^{1,2)}$. In particular, heat-induced gelation materials, which undergo a thermally reversible transition from a low viscosity solution to a rigid gel upon an increase in temperature, have attracted considerable attention as injectable drugdelivery systems ${ }^{3)}$. The most notable heat-induced gelator is the cross-linked poly (N-isopropylacrylamide) (PNIPAM) hydrogel, which exhibits a volume phase transition upon heating through its lower critical-solution temperature $(\text { LCST })^{4)}$. However, cross-linked PNIPAM and its copolymers have limited flexibility and injectability. Thus, it is desirable to develop high-performance heat-induced gelators without chemical cross-links.

Supramolecular gelation systems which exhibit normal gelation behavior without chemical cross-links have been reported in various structures of low-molecular weight compounds $^{5-7)}$; however, reports on heat-induced gels formed from low-molecular weight compounds remain limited. Tobita et $a l^{8}{ }^{8}$ showed that the viscosity of a fluorohybrid-type surfactant solution increased gradually over a wide temperature range of $10-36{ }^{\circ} \mathrm{C}$, with a maximum viscosity at $36^{\circ} \mathrm{C}$, which decreased upon further heating. Similar viscosity curves upon heating have also been observed in other surfactant solutions ${ }^{8,9)}$. In the heat-induced gelation of surfactants, the phase transitions generally occur over a broad temperature range and the surfactant solutions exist as a viscous liquid at maximum viscosity rather than a gel.

Recently, we demonstrated that a long-chain amidoamine derivative (C18AA; Fig. 1 ${ }^{10,11)}$ acted as a normal organogelator in various organic solvents. Further, we reported another important finding that $\mathrm{O} / \mathrm{W}$ emulsions formed from a toluene/C18AA/aqueous LiCl system have novel heat-induced gel properties. In the report, $\mathrm{LiCl}$ was found to be essential for the formation of the heat-induced gel, but it remained an unsettled question whether com-

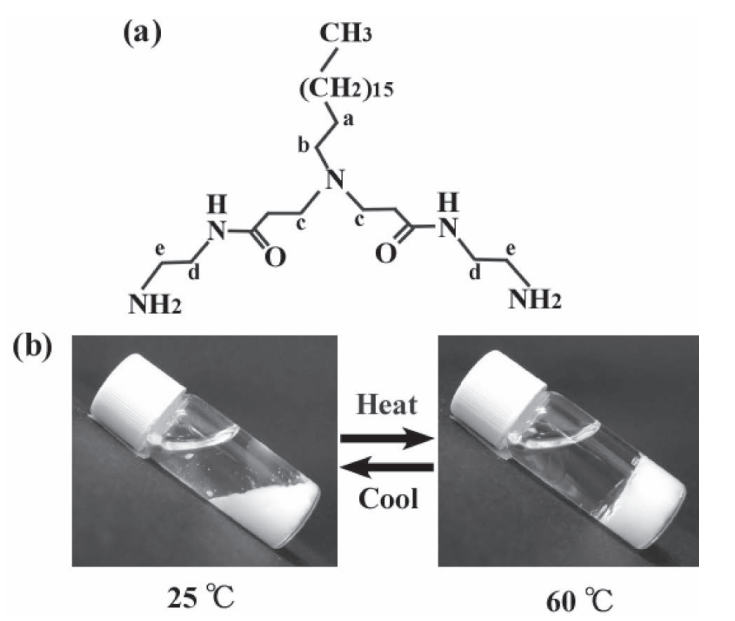

Fig. 1 (a) Molecular structure of C18AA and (b) photographs of heat-induced gel of $1.0 \mathrm{wt} \%$-C18AA/ toluene/ $0.12 \mathrm{M} \mathrm{HCl}$ upon heating and cooling.

\footnotetext{
*Correspondence to: Takeshi Kawai, Department of Industrial Chemistry, Tokyo University of Science, 1-3 Kagurazaka, Shinjuku-ku, Tokyo 162-8601, JAPAN

E-mail: kawai@ci.kagu.tus.ac.jp

Accepted June 17, 2011 (received for review May 23, 2011)

Journal of Oleo Science ISSN 1345-8957 print / ISSN 1347-3352 online

http://www.jstage.jst.go.jp/browse/jos/ http://mc.manusriptcentral.com/jjocs
} 
pounds apart from $\mathrm{LiCl}$ would be effective in forming $\mathrm{O} / \mathrm{W}$ emulsions of C18AA to act as heat-induced gels. Moreover, the reversible sol-gel transition of heat-induced gels produced by a $\mathrm{LiCl}$ electrolyte cannot be regulated by electrolyte concentration, because there is no way to achieve a decrease in concentration.

However, control of the sol-gel transition of O/W emulsions of C18AA by $\mathrm{pH}$ would provide a double-stimuli responsive gel. That is, a sol-gel phase transition of the gel could be reversibly regulated by both $\mathrm{pH}$ and temperature. In the present paper, we report on heat-induced gels from O/W emulsions comprising C18AA, hydrochloric acid, and toluene, in which the sol-gel transition can also be reversibly regulated by $\mathrm{pH}$.

\section{EXPERIMENTAL PROCEDURES}

\subsection{Materials}

All chemicals were reagent grade and obtained from Aldrich or Kanto chemicals. Commercially available reagents and solvent were used without further purification, except methyl acrylate (Kanto chemicals), which was purified by distillation under reduced pressure in a nitrogen atmosphere, and octadecylamine, which was recrystallized two times from hexane.

\subsection{Preparation of stimuli-responsive gel}

C18AA was synthesized according to a previous paper ${ }^{10,11)}$. A typical preparation of the stimuli-responsive gel was as follows. An aqueous solution of $0.12 \mathrm{M}$ hydrochloric acid $(80 \mu \mathrm{L})$ was added to $1.0 \mathrm{wt} \% \mathrm{C} 18 \mathrm{AA}$ toluene solution $(2.4$ $\mathrm{g})$, and the mixture was then vigorously stirred overnight. As a result, the mixture was separated into a clear toluene phase in the upper layer and an opaque water phase in the bottom layer, which possesses the heat-induced gel property.

\subsection{Dynamic rheological measurements}

Dynamic rheological experiments were performed on a Thermo Scientific MARS rheometer. A cone-plate with a diameter of $35 \mathrm{~mm}$ and an angle of $1^{\circ}$ was employed. Measurements of the storage $\left(G^{\prime}\right)$ and loss $\left(G^{\prime \prime}\right)$ moduli to give the complex viscosity $\eta^{*}$ were performed as a function of temperature from 10 to $70^{\circ} \mathrm{C}$ at a constant frequency of 1 $\mathrm{Hz}$ and a sheer stress $\tau$ of $1 \mathrm{~Pa}$. The rate of temperature increase was controlled at $3^{\circ} \mathrm{C} / \mathrm{min}$. The complex viscosity $\eta^{*}$ was obtained from the following equation(1), where $f$ is the frequency of oscillation.

$$
\left.\eta^{*}=\left[G^{2}+G^{\prime \prime 2}\right] /(2 \pi f)^{2}\right]^{1 / 2}
$$

\subsection{The distribution ratio of C18AA in the O/W emulsion}

To determine the distribution of C18AA among water, toluene and the water/toluene interface in the stimuli-responsive gel, we measured the solubility of C18AA in the water and toluene phases. An emulsion of $0.5 \mathrm{wt} \%-\mathrm{C} 18 \mathrm{AA} /$ toluene $(100 \mathrm{~mL}) / 0.12 \mathrm{M}$ hydrochloric acid (4 mL) was prepared. After the $\mathrm{O} / \mathrm{W}$ emulsions underwent creaming at a given temperature, a clear water phase in the bottom layer and an excess transparent toluene phase in the upper layer could be observed. Aliquots of the water and toluene phases were pipetted from each layer to measure the C18AA solubility. The water and toluene were removed by freeze-drying in a vacuum and the remaining C18AA was determined by the residual weight. Here, we assume that the C18AA concentration in the toluene phase in the $\mathrm{O} / \mathrm{W}$ emulsions is equal to that in the excess toluene phase, and that hydrochloric acid is insoluble in the toluene phase.

Thus, the distribution coefficients for C18AA molecules in the water phase, $\phi_{\mathrm{W}}$, and in the toluene oil phase, $\phi_{\mathrm{O}}$, were determined on the basis of the weight method as mentioned above. In addition, the distribution coefficient at the O/W interface, $\phi_{\mathrm{i}}$, was evaluated from $\phi_{\mathrm{W}}+\phi_{\mathrm{i}}+\phi_{\mathrm{O}}=$ $100 \%$.

\subsection{Proton NMR spectroscopy}

To clarify the protonation sites of C18AA at low $\mathrm{pH},{ }^{1} \mathrm{H}$ NMR spectra of deuterium oxide solutions of C18AA were measured as a function of $\mathrm{pH}$ using a Bruker DPX-400 spectrometer. The $\mathrm{pH}$ of the solution was adjusted using deuterium chloride.

In order to investigate the morphology of the molecular assembly of C18AA in the opaque water phase, i.e., to determine the stimuli-responsive gel properties, fluorescence microscopy and differential interference contrast microscopy was performed using a Leica DMI4000B. The watersoluble dye rhodamine $6 \mathrm{G}$ was used for fluorescence microscopy.

\section{RESULTS AND DISCUSSION}

\subsection{Thermal behavior of stimuli-responsive gel of C18AA}

The opaque water phase, which was prepared from 0.12 M hydrochloric acid and 1.0 wt\% C18AA toluene gel, was in a sol state at room temperature, but the water phase became rigid on heating, as shown in Fig. 1b. Interestingly, this remarkable thermo-responsive change in fluidity was completely reversible upon heating and cooling cycles. As a result, the addition of hydrochloric acid resulted in a "heat-induced gel" with the opposite thermal property to that of the normal gel. Thus, it was found that a heat-induced gel based on C18AA could also be prepared using $\mathrm{HCl}$, in addition to the $\mathrm{LiCl}$ used previously ${ }^{11)}$.

Figure 2 shows plots of the complex viscosity $\left(\eta^{*}\right)$ against temperature at various concentrations of C18AA. The $\eta^{*}$ values sharply increased at the sol-gel phase transi- 
tion. The thermal response of the heat-induced gel of C18AA was highly sensitive, and the sol-gel transition was completed over a narrow temperature range of $\sim 3^{\circ} \mathrm{C}$. The sol-gel transition temperatures decreased with increasing C18AA concentration. Interestingly, the transition temperatures decreased linearly with increasing C18AA concentration from 0.6 wt \% to 1.4 wt\% (Fig. 3). The existence of a linear relation between the transition temperature and the $\mathrm{C} 18 \mathrm{AA}$ concentration over a wide temperature range of $5-55^{\circ} \mathrm{C}$ in Fig. 3 indicates that the sol-gel transition temperature can be controlled simply by changing the C18AA concentration. In addition, the $\eta^{*}$ values in the sol and gel states were $10^{-1} \mathrm{~Pa} \cdot \mathrm{s}$ and $10^{3} \mathrm{~Pa} \cdot \mathrm{s}$, respectively, irrespective of the $\mathrm{C} 18 \mathrm{AA}$ concentration; that is, the C18AA concentration affected the transition temperature, but not the rigidity of the gel. This provides opportunities for potential

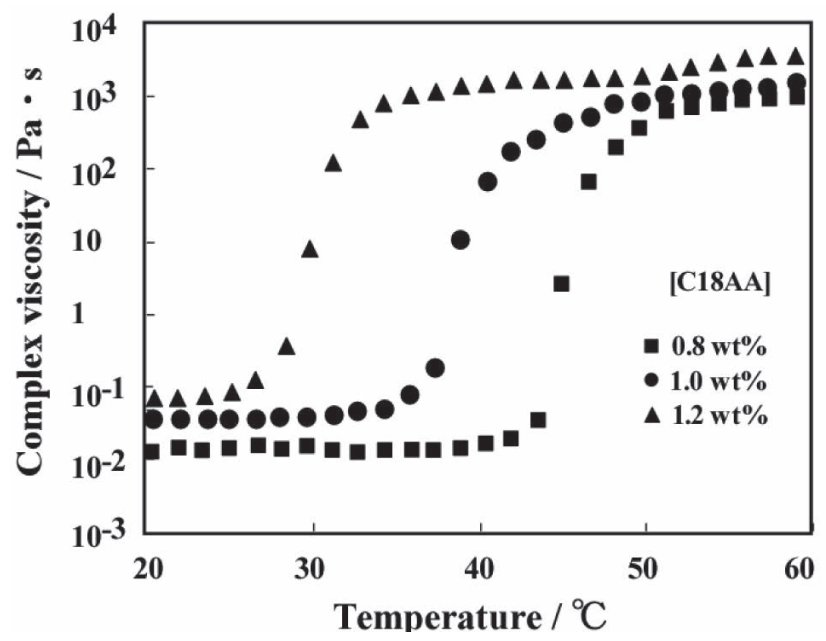

Fig. 2 Temperature dependence of $\eta^{*}$. of C18AA/toluene $/ 0.12 \mathrm{M} \mathrm{HCl}$ at various concentrations of C18AA.

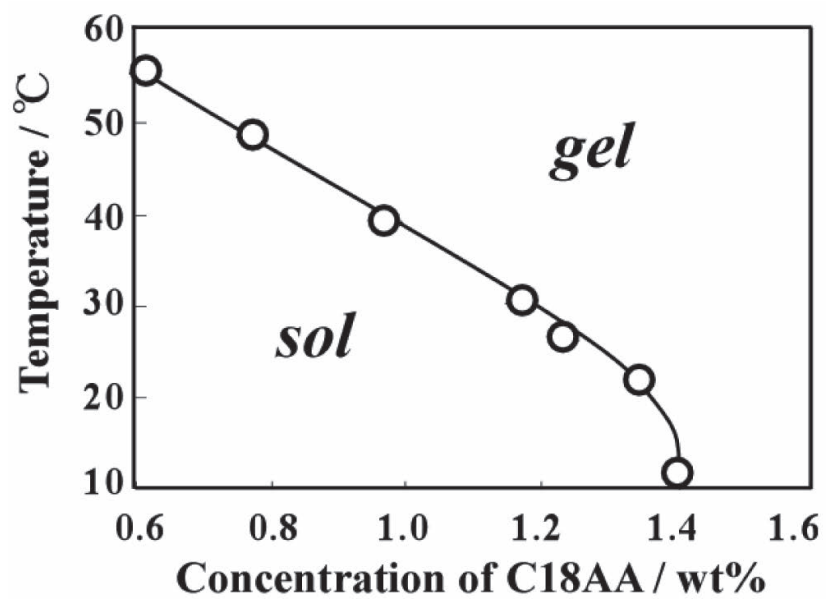

Fig. 3 Sol-gel phase diagram of C18AA/toluene/0.12 $\mathrm{M} \mathrm{HCl}$. application in useful new stimuli-responsive materials.

\subsection{Mechanism of sol-gel phase transition}

In order to investigate differences in the emulsion structure between the sol and gel states, we obtained optical microscopic images of the opaque phases in the sol and gel states. Figure 4 shows the interference contrast images (left) and fluorescence images (right) of C18AA concentrations of $0.8 \mathrm{wt} \%$ (a) and $1.3 \mathrm{wt} \%$ (b) at room temperature, corresponding to the sol and gel states, respectively. The opaque phases in both the sol and gel states consisted of emulsion droplets with diameters of 1-50 $\mu \mathrm{m}$. At the sol-gel phase transition, the droplets changed from a spherical to a non-spherical shape, however, both emulsions basically consisted of O/W types ${ }^{12)}$, as confirmed by fluorescence images obtained using the water soluble dye rhodamine $6 \mathrm{G}$ (Fig. 4).

The emulsion structure did not differ much between the sol and gel states, and both phases consisted of O/W emulsions. Next we examined the distribution of C18AA among water, toluene and the water/toluene interface in a 0.5 wt\% C18AA/toluene /0.12 M HCl system as a function of temperature. Figure 5 reveals that the adsorption density of C18AA at the water/toluene interface decreases with increasing temperature. In other words, increasing the temperature results in a transfer of C18AA molecules from the $\mathrm{O} / \mathrm{W}$ interface to the continuous water phase. Thus, deformation of the droplets at a higher temperature is caused by

(a)

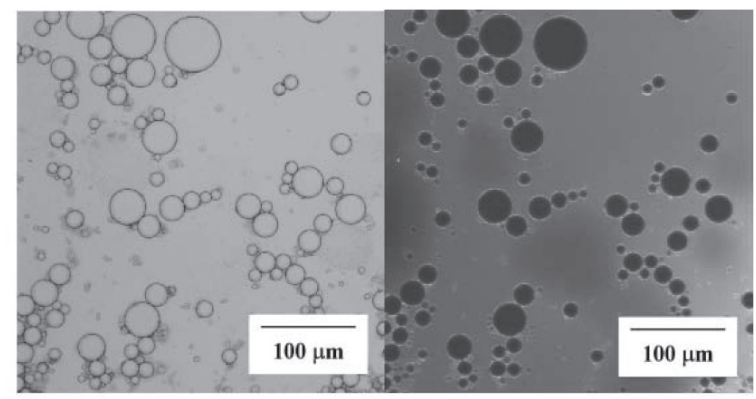

(b)

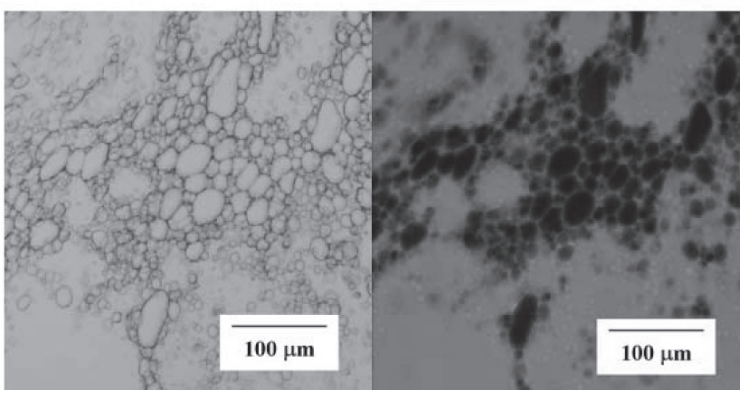

Fig. 4 Images from differential interference contrast microscopy (left) and fluorescence microscopy (right) at room temperature of (a) $0.8 \mathrm{wt} \%$ C18AA/toluene/0.12 M HCl (sol state) and (b) $1.3 \mathrm{wt} \%$-C18AA/toluene $/ 0.12 \mathrm{M} \mathrm{HCl}$ (gel state). 


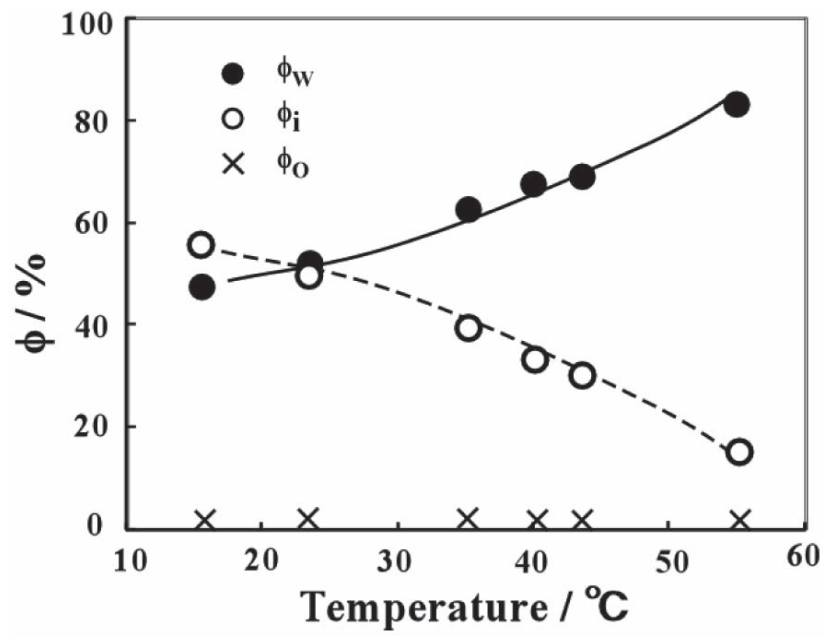

Fig. 5 Temperature dependence of the distribution of C18AA in toluene $/ 0.12 \mathrm{M} \mathrm{HCl}$. The indices inidicated the distribution coefficients for C18AA molecules in the water phase, $\phi_{\mathrm{W}}$, in the toluene oil phase, $\phi_{\mathrm{O}}$, and at the $\mathrm{O} / \mathrm{W}$ interface, $\phi_{\mathrm{i}}$. $\left(\phi_{\mathrm{W}}+\right.$ $\phi_{\mathrm{i}}+\phi_{\mathrm{O}}=100 \%$.)

a decrease in adsorption of C18AA at the interface.

Significantly, although the droplets did deform to a nonspherical shape at higher temperatures, no phase separation was observed for a month in this system. In general, when emulsions become unstable due to a change in external conditions, phase separation sets in. Thus, the anomalous stability of the emulsions at high temperatures indicates the formation of a network structure within the continuous water phase. In previous work ${ }^{11)}$, we showed that larger aggregates of C18AA were formed in the water phase at higher concentrations. Thus, O/W emulsions in the gel state are trapped and pinned by the network of C18AA aggregates formed in the continuous water phase, leading to a high viscosity for the system (Fig. 6). Consequently, the mechanism of the sol-gel phase transition in the $\mathrm{HCl} / \mathrm{C} 18 \mathrm{AA} /$ toluene system was the same as that in the $\mathrm{LiCl} / \mathrm{C} 18 \mathrm{AA} /$ toluene system previously reported ${ }^{11)}$.

\section{3 $\mathrm{pH}$-responsive behavior of C18AA gel}

In order to reveal the effect of $\mathrm{HCl}$ on the viscosity behavior, $\eta^{*}$ values were measured for the $1.0 \mathrm{wt} \% \mathrm{C} 18 \mathrm{AA}$ systems at various $\mathrm{HCl}$ concentrations on heating (Fig. 7). We found that the $\eta^{*}$ value at the gel state increased with increasing $\mathrm{HCl}$ concentration. The $\eta^{*}$ values for $[\mathrm{HCl}]=0$ M remained comparatively low at $55^{\circ} \mathrm{C}$, whereas the $\eta^{*}$ values for $[\mathrm{HCl}]=0.12 \mathrm{M}$ were extensively high, reaching a maximum of $\sim 10^{3} \mathrm{~Pa} \cdot$ s, i.e. tenfold value of $\eta^{*}$ at $[\mathrm{HCl}]=$ 0 , with transition to a gel occurring at a high temperature. For concentrations up to $[\mathrm{HCl}] \approx 0.12 \mathrm{M}$, the sol-gel phase transition temperatures decreased with increasing $\mathrm{HCl}$ concentration. Interestingly, at $[\mathrm{HCl}]=0.2 \mathrm{M}$ no sol-gel
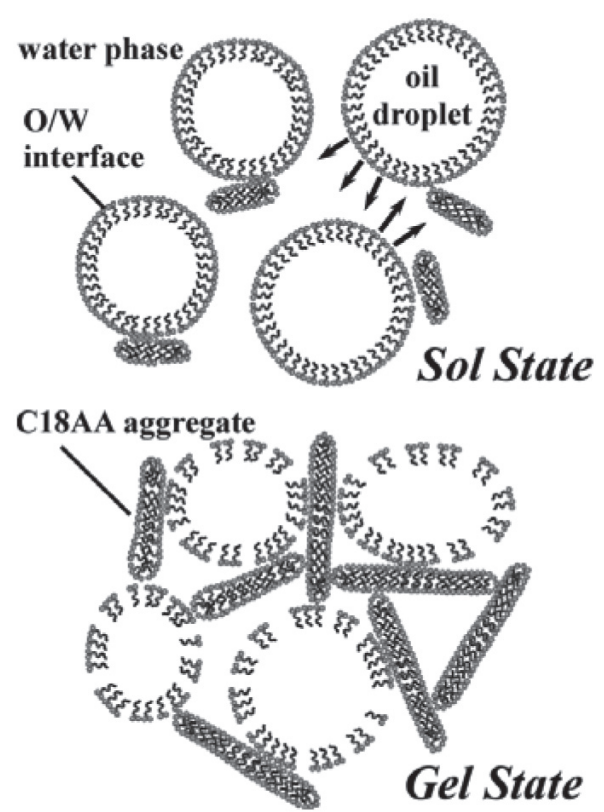

Fig. 6 Schematic illustration of the proposed mechanism for gelation of $\mathrm{C} 18 \mathrm{AA} /$ toluene/ $\mathrm{HCl}$ aq.

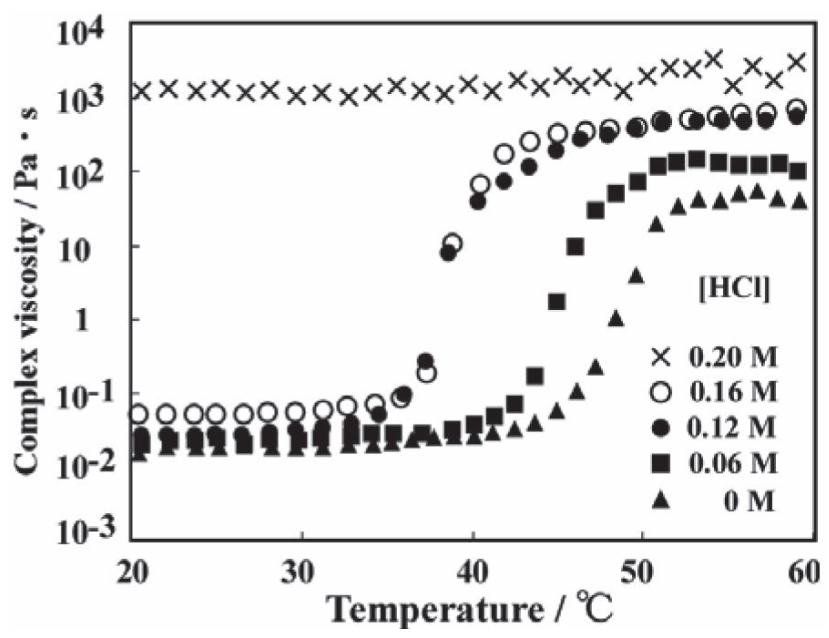

Fig. 7 Temperature dependence of $\eta^{*}$ of $1.0 \mathrm{wt} \%$ $\mathrm{C} 18 \mathrm{AA} /$ toluene $/ \mathrm{HCl}$ at various $\mathrm{HCl}$ concentrations.

transition was observed and the emulsion was in a gel state even at low temperatures.

We then examined the effect of $\mathrm{pH}$ on the sol-gel phase transition temperatures, instead of the nominal $\mathrm{HCl}$ concentration. Figure 8 shows the resultant phase diagram of the heat-induced gel for a $1.0 \mathrm{wt} \% \mathrm{C} 18 \mathrm{AA} /$ toluene/HCl aq. system. At $\mathrm{pH}>9.5$, the phase transition temperatures increase with increasing $\mathrm{pH}$, while at $\mathrm{pH}<9.3$ the system exist in a gel state regardless of temperature. The phase diagram indicates that the sol-gel phase transition can be controlled by $\mathrm{pH}$, as well as temperature. As shown in Fig. 9 , we successfully controlled the totally reversible sol-gel 


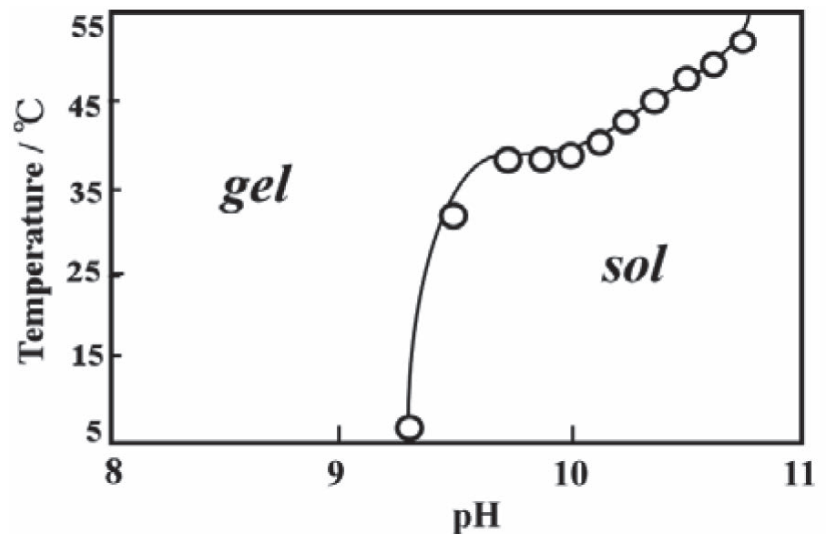

Fig. 8 Sol-gel phase diagram of $1.0 \mathrm{wt} \%$-C18AA/toluene/HCl.
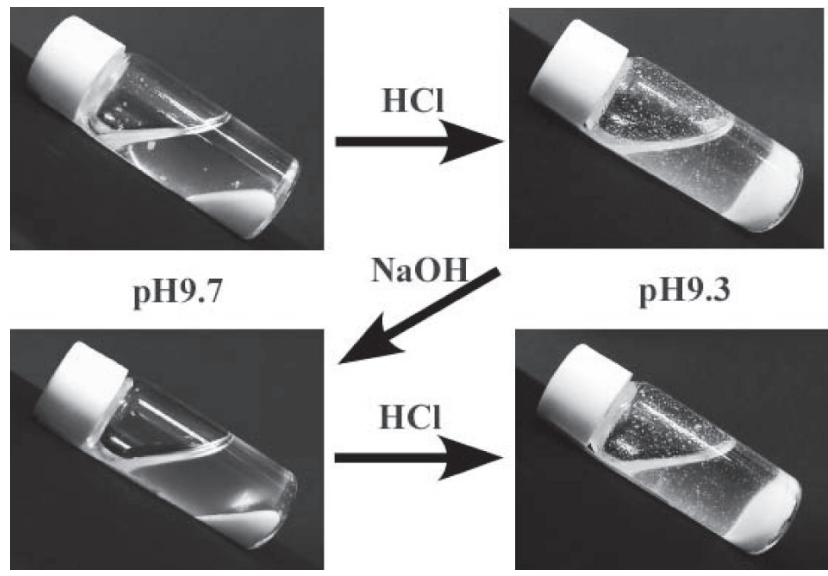

pH9.3

Fig. 9 Photographs of $\mathrm{pH}$-sensitive sol-gel transition of C18AA/toluene/HCl.

phase transition by changes in pH over many cycles. Importantly, the sol-gel phase transition showed a highly sensitive $\mathrm{pH}$ response, and the $\mathrm{pH}$ range of the transition between the sol and gel states was pH 9.5 \pm 0.2 .

It is well known that the terminal and tertiary amine groups of $\mathrm{C} 18 \mathrm{AA}$ act as protonation sites at a lower $\mathrm{pH}$. Thus, the pH-responsive sol-gel transition in Fig. 8 is probably related to protonation of the amines. Since protonation would lead to variation of the ${ }^{1} \mathrm{H}$ chemical shifts of methylene groups adjacent to the protonated sites ${ }^{13-15)}$, obtaining plots of these chemical shifts against $\mathrm{pH}$ can provide detailed information on the protonation degree. Figure 10 shows the chemical shifts of several methylene groups as a function of $\mathrm{pH}$. In the $\mathrm{pH}$ range of $6-12$, the chemical shifts of the methylene groups adjacent to the tertiary amine group were unchanged; however, below $\mathrm{pH}$ $=\sim 9.5$ those of the methylene groups adjacent to the terminal amine groups were shifted to lower field. This result indicates that at $\mathrm{pH}>9.5$ there is no protonation of either amine group, while at $\mathrm{pH}<9.5$ the terminal amine groups start to protonate. (a)

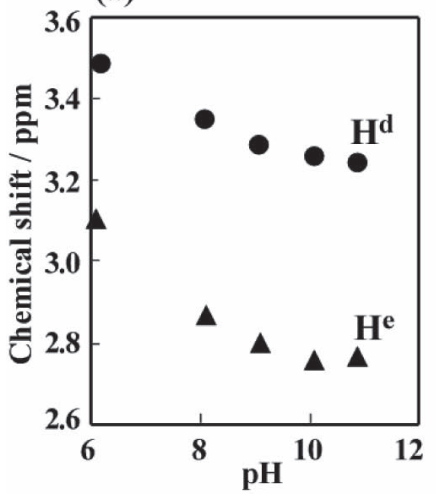

(b)

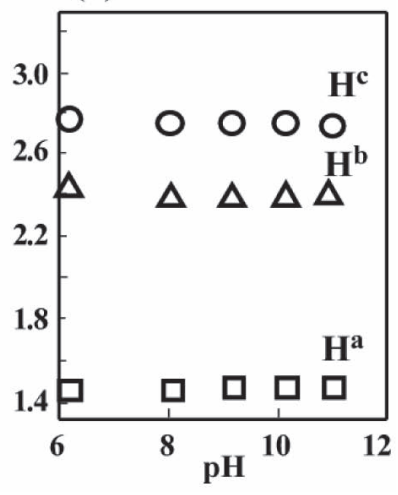

Fig. $10{ }^{1} \mathrm{H}$ NMR-pH titration plots of C18AA. The indices of a e indicate the corresponding proton position shown in Fig. 1.

Thus, the gradual decrease in sol-gel phase transition temperature with decreasing $\mathrm{pH}$ above $\mathrm{pH}=\sim 9.5$ observed in Fig. 8 is not caused by protonation of C18AA, but by a salting-in effect ${ }^{16)}$ of $\mathrm{H}^{+}$, which acts as a chaotropic cation ${ }^{17)}$, in the same way as $\mathrm{Li}^{+}$in the previous system ${ }^{11}$. On the other hand, the abruptly decrease in sol-gel transition with decreasing $\mathrm{pH}$ below $\mathrm{pH}=9.5$ is directly related to protonation of the terminal amine groups. The protonated C18AA molecules are more soluble in water according to their higher hydrophilicity. As a consequence, decreasing the $\mathrm{pH}$ results in a transfer of C18AA molecules from the $\mathrm{O} / \mathrm{W}$ interface to the continuous water phase, and subsequent formation of larger aggregates of C18AA in the water phase. The O/W emulsions are trapped and pinned by the network of C18AA aggregates formed in the continuous water phase, leading to gel formation. The mechanism of the sol-gel phase transition regulated by a change in $\mathrm{pH}$ is the same as that occurring by a change in temperature, as mentioned in the above section. Thus, it was demonstrated that sol-gel transition of $\mathrm{O} / \mathrm{W}$ emulsions could be regulated reversibly by a change in $\mathrm{pH}$, in addition to temperature, thereby providing an $\mathrm{O} / \mathrm{W}$ emulsion gel based on $\mathrm{C} 18 \mathrm{AA}$ and $\mathrm{HCl}$ that was double-stimuli responsive, that is, thermo- and pH-responsive.

\section{CONCLUSION}

We have demonstrated that O/W emulsions of a C18AA/ toluene/hydrochloric acid system can be induced to undergo sol-gel phase transition by a change in temperature or $\mathrm{pH}$, suggesting their potential for thermo-responsive and $\mathrm{pH}$-responsive materials. The $\mathrm{O} / \mathrm{W}$ emulsion gel acted as a heat-induced gel with opposite thermal property to that of a normal gel and underwent a phase transition from sol to gel on heating. Further, it was possible to reversibly control the sol-gel phase transition by a change in 
pH over many cycles. Importantly, the sol-gel phase transition showed a highly sensitive $\mathrm{pH}$ response, and the $\mathrm{pH}$ range between the sol and gel states was quite narrow.

\section{ACKNOWLEDGEMENT}

This work was partially supported by a Giant-in-Aid for Scientific Research B from the Ministry of Education, Culture, Sports, Science and Technology of Japan.

\section{References}

1) Beebe, D. J.; Moore, J. S.; Bauer, J. M.; Yu, Q.; Liu, R. H.; Devadoss, C.; Jo, B.-H. Functional hydrogel structures for autonomous flow control inside microfluidic channels. Nature 404, 588(2000).

2) Kiyonaka, S.; Sugiyama, K.; Shinkai, S.; Hamachi, I. First thermally responsive supramolecular polymer based on glycosylated amino acid. J. Am. Chem. Soc. 124, 10954 (2002).

3) Li, Z.; Wang, F.; Roy, S.; Sen, C. K.; Guan, J. Injectable, highly flexible, and thermosensitive hydrogels capable of delivering superoxide dismutase. Biomacromolecules 10, 3306 (2009).

4) Zhang, Y.; Furyk, S.; Bergbreiter, D. E.; Creamer, P. S. Specific ion effects on the water solubility of macromolecules: PNIPAM and the Hofmeister series. J. Am. Chem. Soc. 127, 14505 (2005).

5) Imura, Y.; Matsue, K.; Sugimoto, H.; Ito, R.; Kondo, T.; Kawai, T. Ambidextrous gel property and pH-responsive sol-gel transition of low molecular mass gelator based on a long-chain amide derivative. Chem. Lett. 38, 778(2009).

6) Suzuki, M.; Nakajima, Y.; Yumoto, M.; Kimura, M.; Shirai, H.; Hanabusa, K. Effects of hydrogen bonding and van der Waals interactions on organogelation using designed low-molecular-weight gelators and gel formation at room temperature. Langmuir 19, 8622 (2003).

7) Terech, P.; Coutin, A.; Ground-Godquin, A. M. Scattering of a crystalline gel network: a new organogel based upon a benzohydroxamic acid derivative. J. Phys.
Chem. B 101, 6810 (1997).

8) Tobita, K.; Sakai, H.; Kondo, Y.; Yoshino, N.; Iwahashi, M.; Momozawa, N.; Abe, M. Thermoresponsive viscoelasticity of sodium 1-oxo-1-[4-(tridecafluorohexyl) phenyl]2-hexanesulfonate aqueous solutions. Langmuir 13, 5054 (1997).

9) Sharma, S. C.; Rodriguez-Abreu, C.; Shrestha, L. K.; Aramaki, K. Oil-induced anomalous thermoresponsive viscoelasticity in fluorinated surfactant systems. $J$. Phys. Chem. B 111, 12146 (2007).

10) Imura, Y.; Morita, C.; Endo, H.; Kondo, T.; Kawai, T. Reversible phase transfer and fractionation of $\mathrm{Au}$ nanoparticles by $\mathrm{pH}$ change. Chem. Commun. 46, 9206 (2010).

11) Morita, C.; Sugimoto, H.; Matsue, K.; Kondo, T.; Imura, Y.; Kawai, T. Changes in viscosity behavior from a normal organogelator to a heat-induced gelator for a longchain amidoamine derivative. Chem. Commun. 46, 7969 (2010).

12) Sun, G.; Li, Z.; Ngai, T. Inversion of particle-stabilized emulsions to form high-internal-phase emulsions. Angew. Chem. Int. Ed. 49, $2163(2010)$.

13) Koper, G. J. M.; Duijvenbode, R. C.; Stam, D. D. P. W.; Steuerle, U.; Borkovec, M. Synthesis and protonation behavior of comblike poly (ethyleneimine). Macromolecules 36, 2500 (2003).

14) Szakacs, Z.; Beni, S.; Varga, Z.; Orfi, L.; Keri, G.; Noszal, B. Acid-base profiling of Imatinib (Gleevec) and its fragments. J. Med. Chem. 48, 249 (2005).

15) Koper, G. J. M.; Genderen, M. H. P.; Elissen-Roman, C.; Baars, M. W. P. L.; Meijer, E. W.; Borkovec, M. Protonation mechanism of poly (propylene imine) dendrimers and some associated oligo amines. J. Am. Chem. Soc. 119, 6512 (1997).

16) Magnusson, J. P.; Khan, A.; Pasparakis, G.; Saeed, A. O.; Wang, W.; Alexander, C. Ion-sensitive "isothermal" responsive polymers prepared in water. J. Am. Chem. Soc. 130, 10852 (2008).

17) Marcus, Y. A simple empirical model describing the thermodynamics of hydration of ions of widely varying charges, sizes, and shapes. Biophys. Chem. 51, 111 (1994). 